\title{
Performance and Noise Trade-Offs on a Civil Airliner with Over-the-Wing Engines
}

\author{
Stephen Powell*, András Sóbester† Phillip Joseph ${ }^{\ddagger}$ \\ University of Southampton, Southampton, Hampshire, SO17 1BJ, UK
}

\begin{abstract}
Community noise has become a major consideration in the design of new aircraft. The noise generated by the engines has decreased over the generations to the extent that a whole-airframe approach is required now to achieve further significant reductions. One option is to install the engines over the wings so the airframe reflects the fan noise away from on-the-ground observers. However, in addition to good noise shielding performance, the position of the engine also has to satisfy aerodynamic efficiency criteria. We investigate the sensitivity of aerodynamic and acoustic performance metrics with respect to the positioning of the engine relative to the wing. More specifically, we trade drag computed via Reynolds-Averaged Navier Stokes simulations versus noise shielding performance, obtained experimentally through scale model tests conducted in an anechoic chamber. Surrogate models of both metrics are constructed, enabling their Pareto analysis on the specific case of a modified DLR F6 airframe geometry.
\end{abstract}

\section{Introduction}

Since the advent of the civil jet airliner, aircraft noise has been a significant issue, especially to those living in the close proximity of airports. Although measures such as land planning restrictions have been applied to reduce the effect, the rise in air traffic, as well as public awareness, has increased the importance of designing aircraft that reduce the noise experienced by on-the-ground observers. Fig. 1 shows that the perceived noise level (PNL) has been declining with time, but the improvements made due to the high bypass ratios of the second generation turbofans seem to have reached their limit. Modern improvements in engine and nacelle design have led to improved noise reductions, but the ACARE $2020^{1}$ noise target of reducing the average perceived noise by one half is looking increasingly unlikely using current aircraft configurations.

The potential of aircraft configurations that drastically differ from the current convention has yet to be realised. The apprehension of the public - their willingness to fly on an aircraft that is aesthetically different from convention - as well as the large development costs have hindered many designs. In NASA's Subsonic Fixed Wing Project, where three generations of conceptual designs are to be developed for operation at different stages between now and 2035, a number of advanced concept configurations are to be considered. For each generation the designs will move away from

\footnotetext{
${ }^{*} \mathrm{PhD}$ student, Computational Engineering and Design Group.

${ }^{\dagger}$ Lecturer, Computational Engineering and Design Group, AIAA member.

${ }^{\ddagger}$ Lecturer, Institute of Sound and Vibration Research
} 


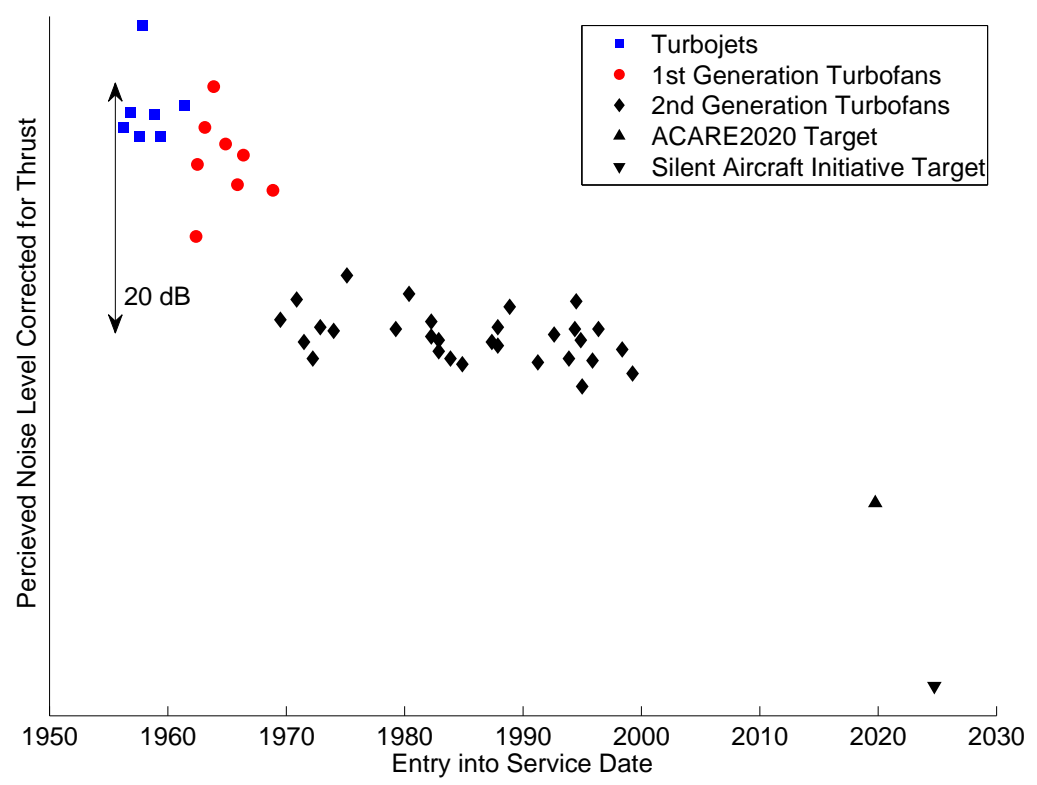

Fig. 1 Reduction of perceived noise level corrected for thrust over time (graph reproduced from Ref. 2)

the convention, with the intention to realise continual reductions in noise, emissions, fuel burn, and field length. For example, an advanced configuration being proposed for generation $\mathrm{N}+3$ (where $\mathrm{N}$ is the configuration in service at this time) is the Blended-Wing-Body(BWB) concept, ${ }^{3}$ which greatly improves aerodynamic efficiency as well as noise reduction.

The first concept $(\mathrm{N}+1)$ of NASA's Subsonic Fixed Wing Project has the general configuration of current designs, using current technologies to reduce noise and improve efficiency. ${ }^{4}$ Investigations to achieve the ambitious goals of the $\mathrm{N}+1$ generation concentrate on the powerplant and its placement, as the general configuration is constrained to remain conventional. Increased powerplant diameter and the use of geared fan drives are expected as side effects of the need to increase efficiency and reduce noise.

The size of the engine is constrained on conventional under-the-wing transports due to its close proximity with the ground. Aspects such as landing gear failure and foreign object ingestion from runways have to be taken into account. Increasing the diameter of engines for a larger bypass ratio (BPR) for improved efficiency can therefore be a difficult integration task. A solution that has been proposed is to install the engines above the wing, therefore alleviating the constraints of the close proximity to the ground.

Many of the powerplant installation constraints listed by Berry ${ }^{5}$ can be met by using over the wing engines, as ground clearance, roll clearance, and passenger escape zones are independent of engine position above the wing. The engine size will only be limited by the load on the wing and attachment fitting limitations. ${ }^{5}$ Kinney $^{6}$ noted that the loss of engine exhaust below the wing would eliminate the need for thrust gates between the flaps, allowing for a single flap that would reduce noise created

2

Submitted to the American Institute of Aeronautics and Astronautics 
by the tip vortices and give larger effective flap area, both of which resulting in aerodynamic benefits. There may however be an exhaust interaction with the upper surface due to the Coanda affect. Other advantages that Kinney identified are the increased flexibility of landing gear placement on the wing, better safety in water ditching and wheels-up landing scenarios, and the benefit of reduced noise due to shielding of the wing that makes this configuration attractive to designers. With the height of the aircraft from the ground independent of the engines, the size of the landing gear only depends on tail scrape angle and the magnitude of rotation at take-off.

The disadvantages of an engine over the wing configuration include maintenance issues with the engine not being accessible from the ground, and a possible increase in cabin noise. ${ }^{7,8}$ A total redesign of support structures in the wing would incur a significant cost when modifying existing designs.

The main benefit, however, of installing an engine over the wing is that the wing acts as a fan noise shield from the perspective of on-the-ground observers. NASA began investigating the advantages of over the wing mounted engines in the early 1970s, in order to reduce aircraft-generated noise affecting communities near airports. ${ }^{9}$ This work was published after the first prototype of the Fokker VFW 614 aircraft took its maiden flight in $1971,{ }^{10}$ the only civil jet airliner to have over the wing mounted engines. The main considerations that led to this design were: the maximum degree of payload flexibility, minimum foreign object matter ingestion, and minimum fuselage ground clearance requirements. Advantages of noise shielding (leading to a 4 EPNdB noise reduction at approach) and undivided landing flap (divided landing flap needed due to engine jet on underwing configurations) were not amongst the main considerations, rather serendipitous effects of the over the wing configuration. Noise shielding was not a primary consideration three decades later for the Honda Business Jet either; the engines were located over the wing as the alternative was to install them on the rear fuselage, which would have reduced the cabin volume due to the structural supports. ${ }^{11}$

Studies by Agarwal et al. ${ }^{12,13}$ have highlighted the noise reducing potential of using parts of the airframe as a shield. Berton ${ }^{7}$ investigated the noise potential of over-the-wing engine installations on conventional aircraft, with promising results. NASA have admitted that an over the wing engine configuration looks promising, and is an area they intend to further explore. ${ }^{14}$ However, a lot of their effort is concentrated to investigating the aft radiated noise on a hybrid wing body (HWB) configuration, ${ }^{15}$ and on noise generated by new engine concepts. ${ }^{16}$ There is an ongoing project studying the open rotor noise installation effects on both HWB and a conventional configuration (see Ref. 15), but to the authors knowledge the results are yet to be published. The results obtained by Yoneta et al. ${ }^{17}$ and Fujino and Kawamura ${ }^{11}$ show that the aerodynamic performance of an engine over-the-wing configuration is highly dependent on the engines placement with reference to the wing surface.

In this paper we investigate the potential of these performance gains from an aerodynamic and an acoustic perspective, using surrogate models of each metric to investigate designs representing a range of possible trade-offs. In order to assess noise shielding performance across a range of relative engine positions (with respect to the wing) we have conducted a series of scale model experiments in the University of Southampton's Large Anechoic Chamber. The aerodynamic performance, characterised by the drag experienced at a constant given lift, is analysed through CFD studies running at cruise conditions and across the same range of engine positions. The surrogate modelling approach allows 
us therefore to estimate the performance of any design within the parameter space defined by the two variables that identify the location of the engine. We use these surrogate models to investigate the optimal solution between the two objectives.

\section{Surrogate Modelling}

Surrogate modelling is a method by which we approximate the underlying mapping that links the inputs we modify to the objective we are optimizing. The computational cost of the surrogate is negligible compared to that of the analysis runs or experimental observations it is built on. The surrogate modelling process begins with the construction of a sampling plan that defines the initial set of training points. After evaluating each design in the sampling plan we fit a surrogate to the responses (function values).

A wide range of surrogate modelling techniques have been developed, many of which are described in Ref. 18. We use the Kriging approach here, of which we now give a brief overview (a more detailed description of the technique is given by Forrester et al. ${ }^{18}$ ).

Suppose we have a set of sample data $\mathbf{X}=\left\{\mathbf{x}_{1}, \mathbf{x}_{2}, \ldots \mathbf{x}_{n}\right\}^{T}$ and we have computed the expensive function outputs for each input set $\mathbf{y}=\left\{y_{1}, y_{2}, \ldots, y_{n}\right\}^{T}$. In Kriging we take the function outputs to be the observed values of a set of random variables

$$
\mathbf{Y}=\left(\begin{array}{c}
Y\left(\mathbf{x}_{1}\right) \\
\vdots \\
Y\left(\mathbf{x}_{n}\right)
\end{array}\right)
$$

Each random variable $Y(\mathbf{x})$ is normally distributed with a mean $\mu$ and variance $\sigma^{2}$. Using two sets of inputs, $\mathbf{x}_{i}$ and $\mathbf{x}_{j}$, we can assume that their function values, $y\left(\mathbf{x}_{i}\right)$ and $y\left(\mathbf{x}_{j}\right)$, will be close together if the distance between inputs $\left|\mathbf{x}_{i}-\mathbf{x}_{j}\right|$ is small. Of course this is also assuming that the function is continuous. This is statistically modelled by the correlation of the two random variables of $\mathbf{x}_{i}$ and $\mathbf{x}_{j}$ using the basis function

$$
\operatorname{Corr}\left[\mathbf{Y}\left(\mathbf{x}_{i}\right), \mathbf{Y}\left(\mathbf{x}_{j}\right)\right]=\exp \left(-\sum_{l=1}^{k} \theta_{l}\left|x_{i_{l}}-x_{j_{l}}\right|^{p_{l}}\right)
$$

If $\mathbf{x}_{i}=\mathbf{x}_{j}$ then the correlation equals one, and if $\left|\mathbf{x}_{i}-\mathbf{x}_{j}\right| \rightarrow \infty$ then the correlation equals zero. The $\theta_{l}$ and $p_{l}$ determine the rate of decrease of the correlation and the smoothness as we move in the $l$ th coordinate direction, respectively. The covariance matrix of $\mathbf{Y}$ can be found by

$$
\operatorname{Cov}(\mathbf{Y})=\sigma^{2} \mathbf{R}
$$

The elements of the correlation matrix $\mathbf{R}$ are the individual results of Equation 2. The values of $\theta_{l}$ and $p_{l}$ are then chosen so our model of the function is the most consistent with the observed function data $\mathbf{y}$. To do this we take natural logarithms of the maximum likelihood function:

$$
\ln (L)=-\frac{n}{2} \ln \left(\sigma^{2}\right)-\frac{1}{2} \ln (|\mathbf{R}|)-\frac{(\mathbf{y}-\mathbf{1} \mu)^{T} \mathbf{R}^{-1}(\mathbf{y}-\mathbf{1} \mu)}{2 \sigma^{2}} .
$$

Submitted to the American Institute of Aeronautics and Astronautics 
The expressions for the optimal values of the mean $\mu$ and variance $\sigma^{2}$ can be obtained by taking derivatives of Equation 4 and setting them to zero. The result is:

$$
\begin{gathered}
\hat{\mu}=\frac{\mathbf{1}^{T} \mathbf{R}^{-1} \mathbf{y}}{\mathbf{1}^{T} \mathbf{R}^{-1} \mathbf{1}} \\
\hat{\sigma}^{2}=\frac{1}{n}(\mathbf{y}-\mathbf{1} \hat{\mu})^{T} \mathbf{R}^{-1}(\mathbf{y}-\mathbf{1} \hat{\mu}) .
\end{gathered}
$$

These optimal conditions can now be substituted back into Equation 4 to create the concentrated log-likelihood function:

$$
\ln (L) \approx-\frac{n}{2} \ln \left(\hat{\sigma}^{2}\right)-\frac{1}{2} \ln (|\mathbf{R}|)
$$

From the derivation, we can see that the concentrated likelihood function is only dependent on the correlation matrix $\mathbf{R}$, which itself is dependent on the hyperparameters $\theta$ and $\mathbf{p}$. To find these parameters we must perform an optimization procedure. Forrester et al. ${ }^{18}$ suggest the use of a global method, as the concentrated likelihood function is very quick to compute and it often features multiple local optima.

To find the kriging prediction at a point $\mathbf{x}$ we use

$$
y(\mathbf{x})=\hat{\mu}+\mathbf{r}^{T} \mathbf{R}^{-1}(\mathbf{y}-\mathbf{1} \hat{\mu}) .
$$

The vector $\mathbf{r}$ contains the correlations between the update point and the points used to produce the Kriging using Equation 2.

\section{Experimental Setup}

Our investigation involves local movement of the nacelle in the chordwise $x$ and vertical $z$ directions with respect to the wing (see Figure 2). The spanwise $y$ position is kept constant.

We have used the same type of sampling of this two-dimensional design space for both the computational flow simulations and the acoustics experiments: 40 space-filling points, 36 of which come from an orthogonal array Latin hypercube, ${ }^{19}$ with the four vertices of the space making up the remainder (see Fig. 3 for a depiction of this layout). The orthogonal array-based hypercube has excellent projective and space-filling properties - it can be shown to maximize the maximin uniformity criterion. ${ }^{20}$ Additionally, here it had the serendipitous advantage of facilitating the repeated repositioning of the experimental rig due to the favourable sequencing of its points. We constrain our search space for the nacelle to be between the leading edge of the wing and $0.3 c_{y}$ ahead of the trailing edge, where $c_{y}$ is the wing chord length at the pylon position. The vertical displacement is limited to being no greater than 1.7 nacelle diameters above the wing.

We use the DLR-F6 airframe model as our baseline. This represents a twin engine civil airliner, and was used in the Drag Prediction Workshops organised by the AIAA. ${ }^{21}$ Its wing has an aspect ratio of 9.5 and a leading edge sweep of $27.1^{\circ}$. The nacelle geometry is that of the CFM-56 engine. 


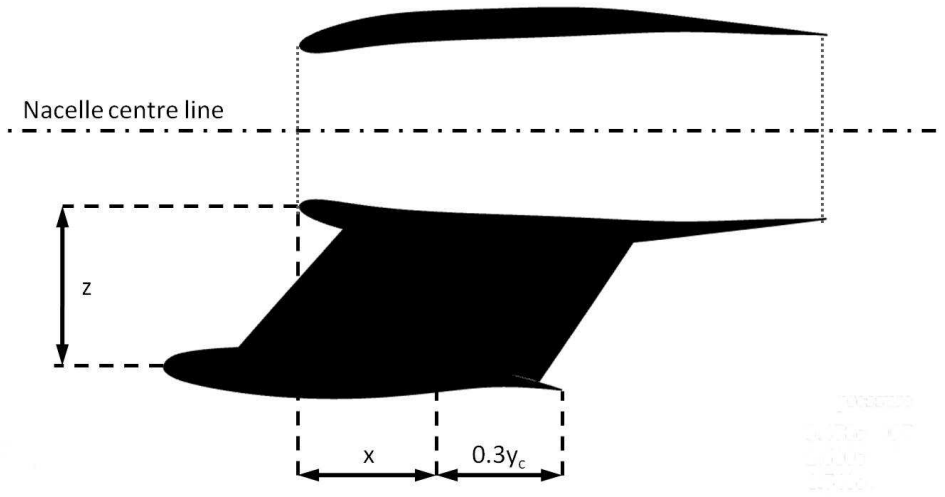

Fig. 2 Diagram of the wing/pylon/nacelle cross sections at their intersections, $\mathrm{x}$ and $\mathrm{z}$ being the design variables of the study described.

\section{Aerodynamic Performance}

\section{Description of Aerodynamic Setup}

The aerodynamic performance in this study is characterised by drag coefficient $\left(C_{D}\right)$ at constant lift coefficient $\left(C_{L}\right)$. We use the same flight conditions as defined for the AIAA drag prediction workshop: ${ }^{21}$ Mach number $M=0.75$ and Reynolds number Re $=5 \times 10^{6}$. Angle of attack was iterated to gain a target $C_{L}$ of 0.5. Flow through nacelles are modelled following from Refs. 17 and 11. The pylon cross section is the symmetrical SC(2)-0012 airfoil, and the pylon movement is constrained by its leading edge interconnection with the nacelle and the wing, and the trailing edge connection with the wing. *

To generate our meshes we use the octree mesher Harpoon. ${ }^{22}$ The geometry of the fuselage and wing is split into sections using CATIA, allowing each section to have a unique mesh size. A similar process is achieved with the pylon and nacelle, but these geometries are generated in MATLAB to provide automatic redefinition of nacelle position and pylon curvature integration with the wing and the nacelle. Stereolithography (STL) files are generated whereby the end points of the pylon STL file are integrated with the wing STL file that was created in CATIA. This improves Harpoon's ability to produce an accurate mesh of this junction. The meshes created have between 7-8 million cells for each model. The Reynolds-Averaged Navier Stokes equations are solved in FLUENT ${ }^{23}$ using the density based implicit solver and the Spalart-Allmaras turbulence model. This setup has been validated against a conventional model from the AIAA drag prediction workshop with acceptable results.

Submitted to the American Institute of Aeronautics and Astronautics 


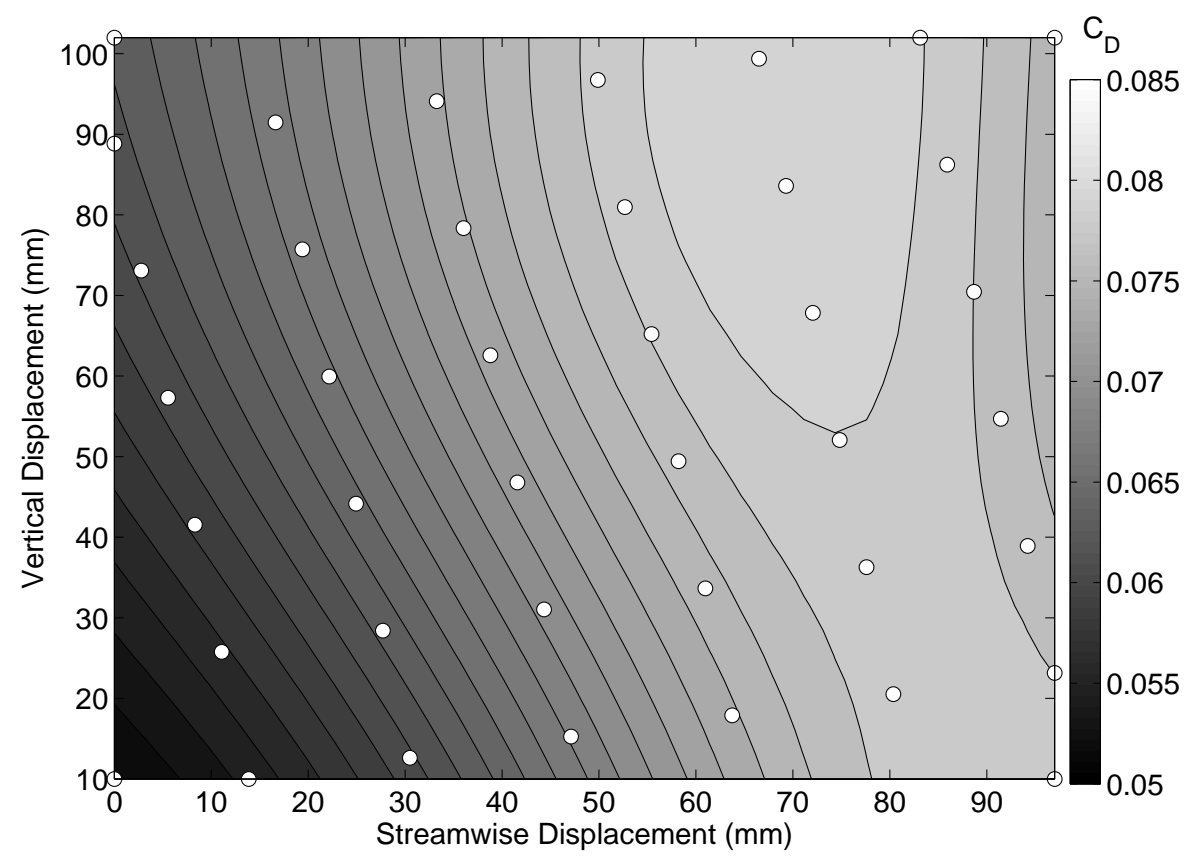

Fig. 3 Surrogate plot of the drag objective, with the white dots indicating the values of the tested designs.

\section{Results}

The CFD simulations were run on the University of Southampton's Iridis 3 supercomputer, which has 1008 Intel Nehalem compute nodes, with each having 22 GB of RAM. Each run used 8 nodes and was run for 5000 iterations, taking just under 28hrs to complete. Fig. 3 shows the Kriging surrogate of the $C_{D}$ objective function for our two parameter design space. The lowest drag was found when the engines were positioned furthest back in our design space, with their vertical position being closest to the wing. At forward positions in the design space the drag values peak and there exists a drag decrease at the furthest forward positions.

It is interesting to assess why we achieve the lowest drag values at certain positions in our design space, mainly to understand what physical features exist that lower the drag values. Of course having an engine installed closer to the wing inherently lowers the profile drag, as less pylon frontal area meets the oncoming flow. Also, moving any geometry near the upper surface (the suction surface) will generally cause some deficiency due to the reduction of lift that it creates. Figs. 4 and 5 show the difference in the surface pressures for the lowest and highest drag cases respectively. Both figures show similar features to those found by Yoneta et al. ${ }^{17}$ There is a large low pressure area inboard of the pylon in Fig 4, with the pylon restricting this low pressure creeping along the span, an effect that was present in the AIAA drag prediction workshop results. ${ }^{24}$ This effect is greater in the high drag case, with Fig. 5 showing a smaller low pressure area in the inboard region. We can also see that

\footnotetext{
*The trailing edge connection with the nacelle is allowed to float so that we obtain the same gradient on the leading and trailing edges of the pylon. This does mean that for some designs the pylon trailing edge finishes aft of the nacelle outlet.
} 
a shock forms outboard of the pylon, approximately in the mid-chord region of the wing. A similar occurrence exists in Fig. 4, but its position is much further aft and thus does not significantly affect the low pressure region on the wing. Another shock occurs in both cases inboard of the pylon, at the pylon/nacelle junction. This is, counter-intuitively greater in the low drag case, but as the shock transfers to the aft region of the wing rather than the mid chord seen in Fig. 5 the efficiency of the wing is not significantly hindered.

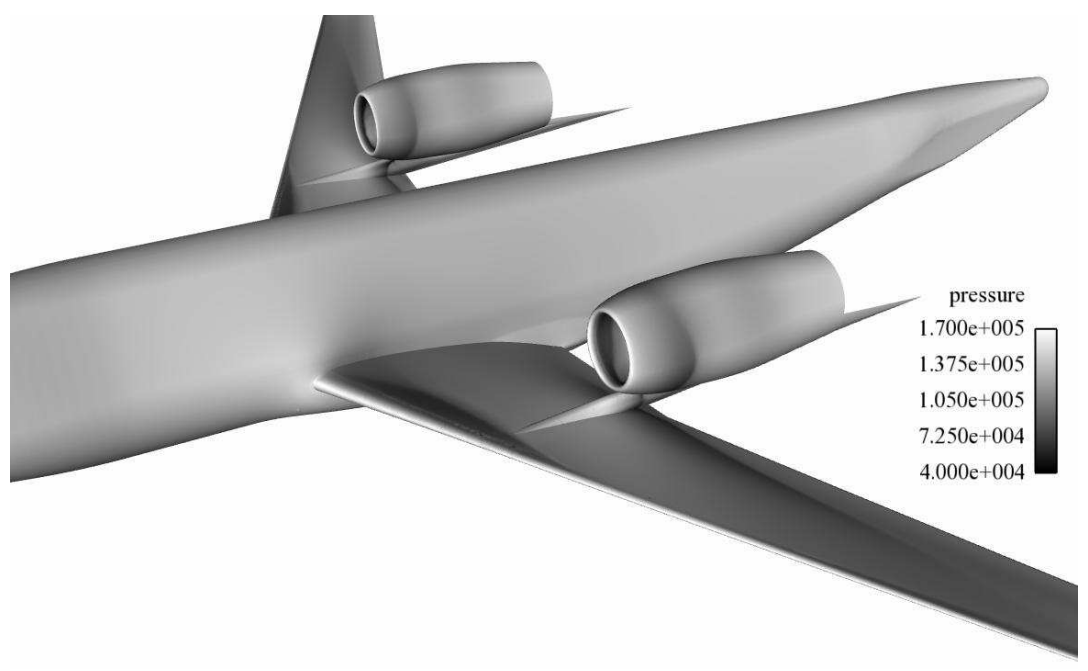

Fig. 4 Pressure contours of the lowest drag case.

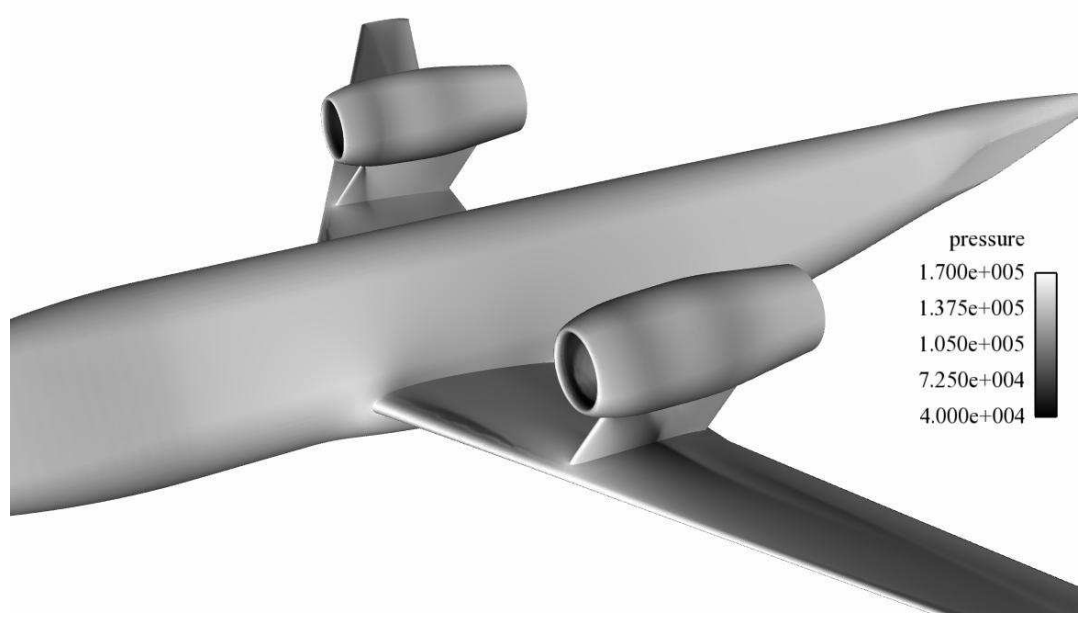

Fig. 5 Pressure contours of the highest drag case. 


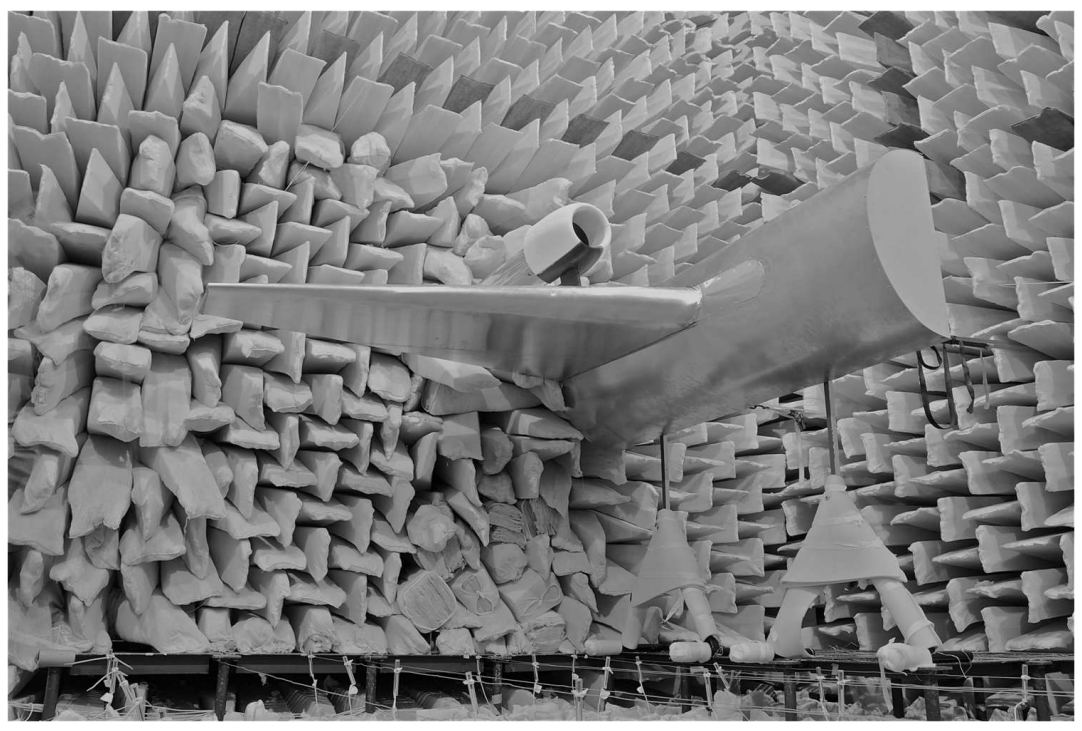

Fig. 6 Setup of our DLR F6 aircraft half model in the University of Southampton's ISVR Large Anechoic Chamber.

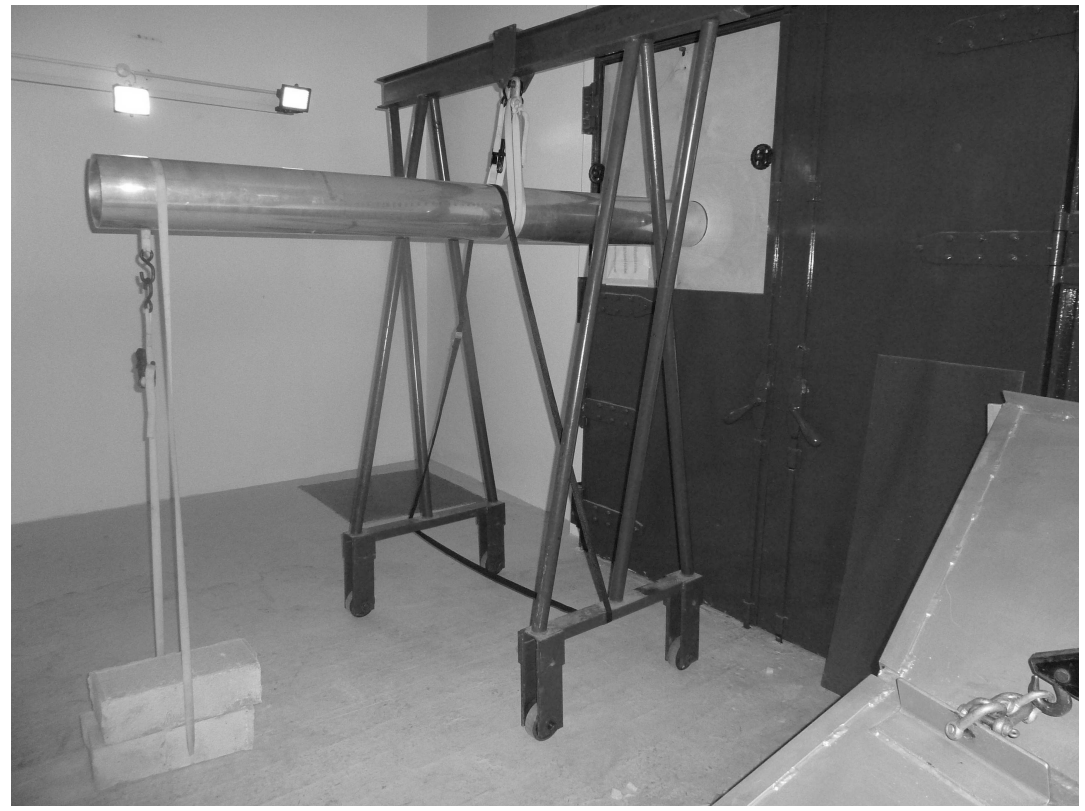

Fig. 7 The reverberation chamber, with the nacelle duct leading into the University of Southampton's ISVR Large Anechoic Chamber.

\section{Noise Shielding Investigation}

\section{Description of the Noise Model}

In order to build a model of the shielding performance of the various propulsion integration geometries considered we devised the experiment depicted in Fig. 6, a photograph of our $12.2 \%^{\dagger}$ DLR $^{-1}$

${ }^{\dagger}$ This is the scale when compared to an Airbus A320 aircraft

9

Submitted to the American Institute of Aeronautics and Astronautics 
F6 half model in the University of Southampton's Large Anechoic Chamber. This model structure is produced from plywood (for the fuselage) and model-quality foam (for the wing), with aluminium sheeting wrapped around to obtain similar noise reflectivity properties to those of a real aircraft. Since we are interested in the fan discharge noise, which is being radiated over the front portion of the wing, we have modified the trailing edge of the wing; removing the kink in the trailing edge slot and increasing the trailing edge thickness to aid manufacturing of the part. The fuselage has a $0.9 \mathrm{~m}$ section ahead of the root leading edge and spans the whole of the root chord. Due to manufacturing constraints, the leading edge sweep had to be altered from $27.1^{0}$ in the design to $25^{0}$. The aircraft model is placed as close to the sound absorbent wedges as possible so secondary noise sources do not emanate off the trailing edge of the wing. We represent the nacelle using an $0.193 \mathrm{~m}$ internal diameter aluminium pipe suspended $1.2 \mathrm{~m}$ above the floor. On the end of the pipe we include a nacelle inlet geometry made from $\mathrm{ABS}^{\ddagger}$ plastic to improve the realism of the model, with a leading portion of the pylon attached to the bottom surface made from the same material. The pipe feeds into the adjacent reverberation room, where it is held in place using ratchet straps. We create different designs by allowing the pipe to move streamwise with reference to the aircraft model. This is achieved using a mobile A-frame structure. Likewise, we achieve vertical displacement by feeding the brackets attached to the flat side of the fuselage through the stand mounts.

In the reverberation chamber (see Fig. 7), a Brüel and Kjaer 1405 noise generator connected to an amplifier and speaker was used to create broadband noise up to $20 \mathrm{kHz}$ (This upper frequency corresponds to a non-dimensional frequency $k a$ of approximately 38 (i.e. between 1 and 2 blade passing frequencies for a modern turbofan), where $k$ is the free space wave number, and $a$ is the duct radius.). Diffuse field excitation of the pipe within the reverberation chamber ensures mutually incoherent modes within the duct and whose amplitudes such that the total acoustic energy is shared equally amongst the modes. ${ }^{25}$ The noise generated is collected by a grid of 20 calibrated microphones positioned on the floor. Once a gain is added using an amplifier, the output is acquired using a National Instruments PXI-1042 data acquisition system at a sampling frequency of 50kHz and fed into a computer for postprocessing. We transform the time signal into the frequency domain using a fast Fourier Transform, correcting for variations in distances for each microphone. We then calculate the overall sound pressure level using the pressure level at each octave band:

$$
\text { OSPL }=20 \log _{10}\left(\frac{\sqrt{\sum_{n=1}^{N} p^{2}}}{2 \times 10^{-5}}\right)
$$

where $p$ is the pressure at octave band, $n$.

Two separate experiments were conducted; one with the aircraft model in the chamber and one with only the nacelle(duct). The amount of shielding is quantified by:

$$
\Delta=20 \log _{10}\left(\frac{\sqrt{\sum_{m=1}^{M}\left(P_{(\text {no aircraft })} / P_{(\text {aircraft })}\right)^{2}}}{2 \times 10^{-5}}\right)
$$

$P$ is the root of the sum of the squares from the individual microphones, and $M$ is the number of

\footnotetext{
$¥$ Acrylonitrile Butadiene Styrene
} 
microphones. We set our design space such that the $(0,0)$ point corresponds to the fully aft position and at the maximum height of the wing surface. Subsequent to the experiment we found that microphone 1 positioned in the far aft end and closest to the fuselage was faulty and therefore its influence has not been recorded in any of the results.

\section{Results}

The experimental study described above yielded a very large amount of data (of the order $24 \mathrm{~Gb}$ ) - here we shall merely summarise its aspects pertaining to the acoustics - aerodynamics trade-offs at the centre of this account. Fig. 8 shows the individual design points and a kriging model acting as a surrogate to the metric. The roughly linear response with streamwise movement of the engine at the furthest forward positions fades as we move the engine backward and vertically closer to the wing, leading to the largest shielding effect at approximately 1.25 nacelle diameters above the wing, in the fully aft position. At streamwise displacements of 0.4 and higher we see a general trend of increased shielding when the engine is closer to the wing and vertical displacement kept constant, with the minimum shielding effect found with the engines $0.07 \mathrm{~m}$ away from the upper surface of the wing at its furthest forward position in the design space. The dependency of the results with regard to the vertical position reduces as we increase the streamwise displacement, which is shown in Fig. 8 by the reduced curvature of the contours as we move the position of the engines further upstream.

To perform comparisons between good and bad designs we have taken the shielding results for each microphone for the best and worst shielding designs and produced a kriging surrogate to map the shielding on the floor (Fig. 9). For the lowest shielding case (case b) we find the contours have a resemblance to the leading edge of the wing, with the best shielding performance achieved under the fuselage. The contours seen in the high shielding case (case a) are significantly different, with most of the shielding achieved ahead of the engine, in front of the leading edge, and not under the planform of the wing. By looking at Fig. 10, which shows the same designs for when the aircraft model is not present in the anechoic chamber, it is clear that the already significant intrinsic shielding effect of the nacelle itself leaves much less room for further improvement in the downstream (aft) section and therefore the improvement in the overall noise footprint of the aircraft is more pronounced forward of the wing leading edge.

For a clear illustration of the shielding performance across the design space and across the physical space of the airframe, we have produced contour plots of the design space for each microphone, shown in Fig. 11. The readings from the microphones positioned away from the fuselage and covered by the wing planform have little or no relationship with the vertical placement of the engine. The variation in this parameter has a greater effect, however, in the intensities recorded on microphones placed away from the wing, due to these microphones being in direct line of sight of the noise source when at the extremities of the design space. The powers measured on the microphones close to the fuselage also have a significant dependence on the vertical position. The plot from microphone 3 shows the lowest shielding being achieved at the furthest forward and lowest position, with the best shielding achieved at the furthest aft streamwise and a high vertical position. In the furthest downstream microphones the lowest shielding remains constant. However, the maximum shielding position gradually moves 


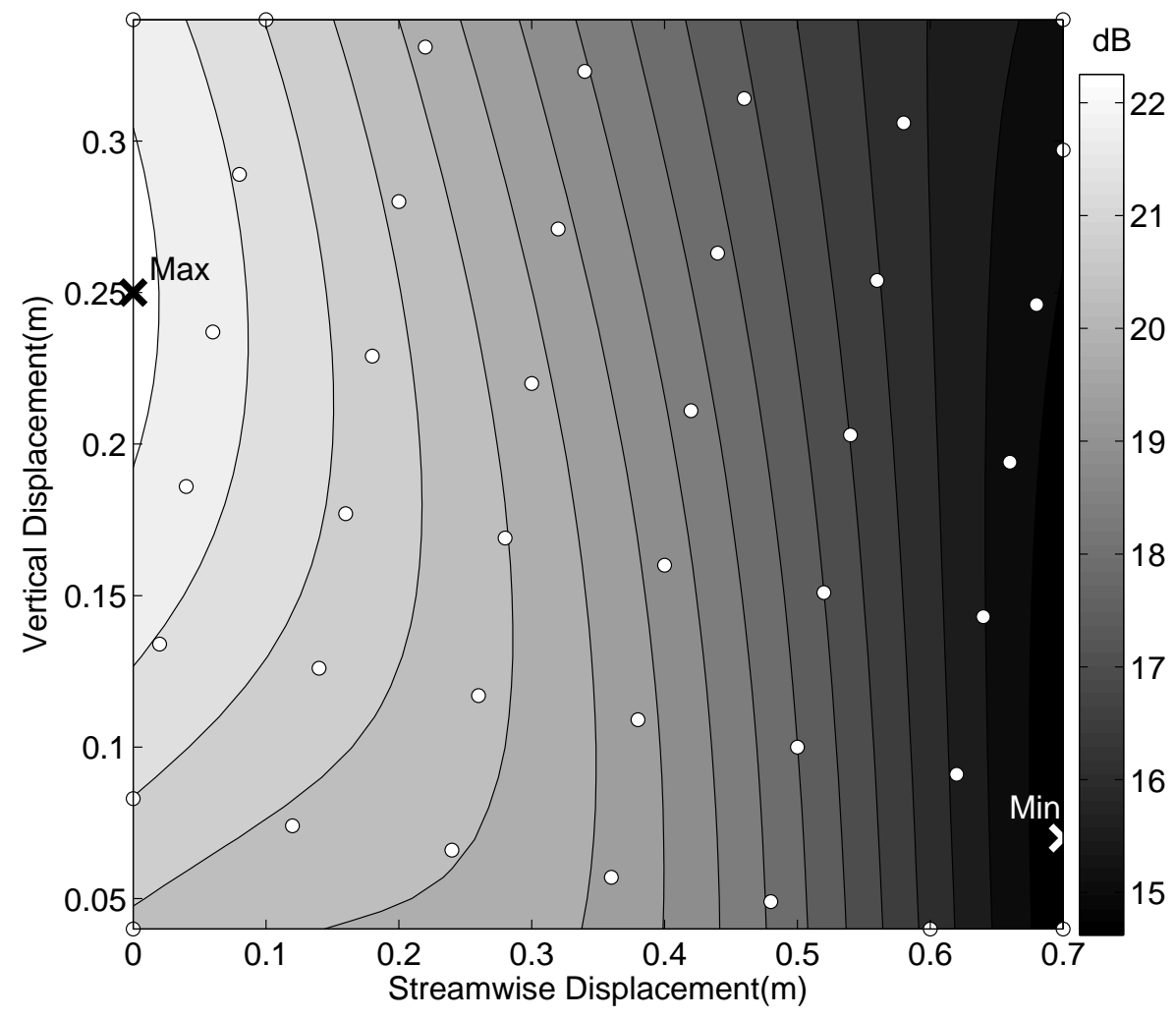

Fig. 8 Kriging prediction of the noise objective in our design space, with the white dots being our experimental designs, and the minimum and maximum predicted points indicated.

from the highest vertical position and furthest back at microphone 5 to the lowest vertical position and furthest back at microphone 20. This may be due to the sweep of the leading edge, making microphone 20 less shielded at higher vertical positions than microphones 5,10, and 15 .

\section{The Trade-offs between Aerodynamic and Noise Performance}

In situations where we can give a weighting factor to the importance of each objective we can effectively run an optimization algorithm on a global objective function that is the linear sum of the single objectives multiplied by their respective weightings. However, in this case study we have no indication of the importance of either objective in relation to each other and thus we build a Pareto set of designs; in a Pareto set each member is non-dominated, which means that no other design can improve on the performance for all objectives. We can view the Pareto set by plotting against each objective function, from which a designer can visually asses which design to be considered the most appropriate.

The Pareto front shown in Fig. 12 was generated using the Non-dominated Sorting Genetic Algorithm (NSGA) II method devised by Deb et al. ${ }^{26}$ This uses a genetic algorithm (GA) to search for the non-dominated designs. The non-dominated solutions at each generation are given a rank of

12

Submitted to the American Institute of Aeronautics and Astronautics 


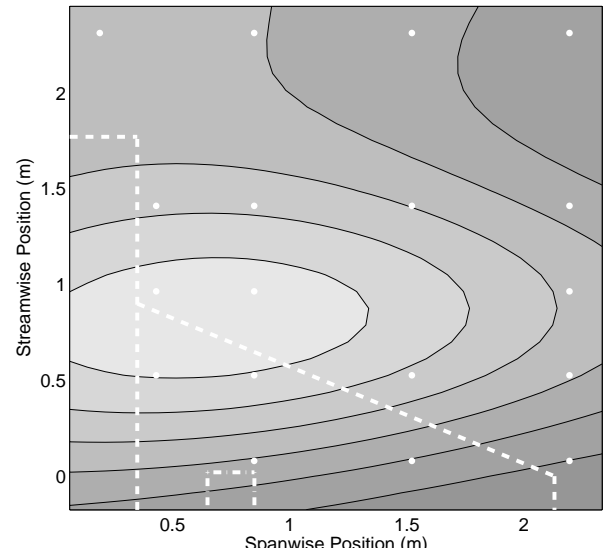

a) Streamwise Position $=0.02 \mathrm{~m}$; Vertical Position $=0.134 \mathrm{~m}$

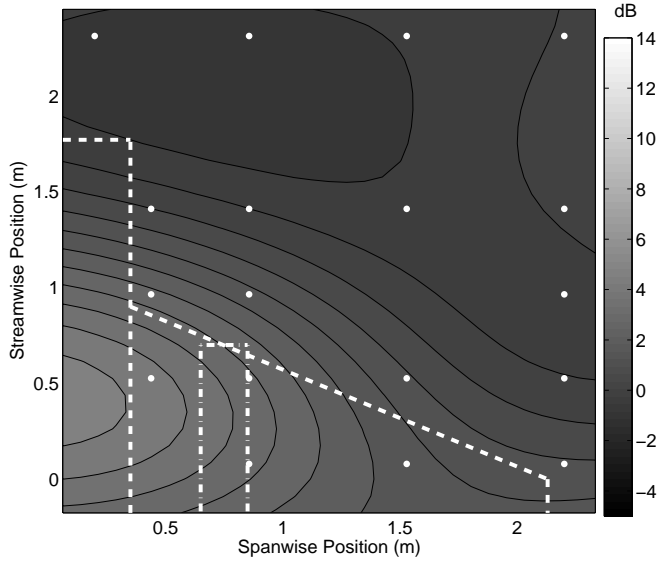

b) Streamwise Position $=0.7 \mathrm{~m}$; Vertical Position $=0.04 \mathrm{~m}$

Fig. 9 Kriging map of the noise objective underneath the a) best and b) worst designs, with the dashed lines indicating the position of the aircraft model and nacelle.

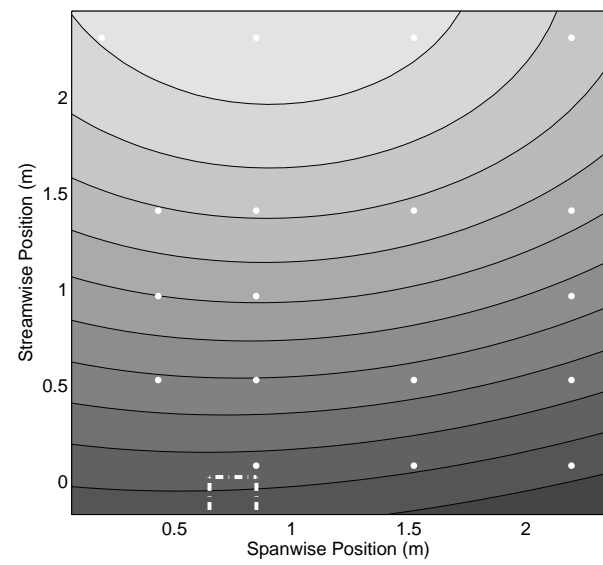

a) Streamwise Position $=0.02 \mathrm{~m}$; Vertical Position $=0.134 \mathrm{~m}$

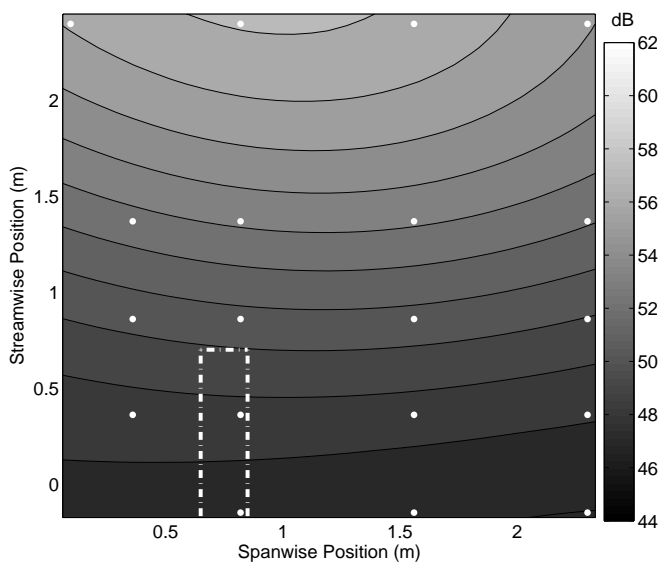

b) Streamwise Position $=0.7 \mathrm{~m}$; Vertical Position $=0.04 \mathrm{~m}$

Fig. 10 Kriging map of the sound pressure level underneath the a) best and b) worst designs when the aircraft model is not present, with the dashed lines indicating the position of the nacelle. 
$\operatorname{mic} 5$
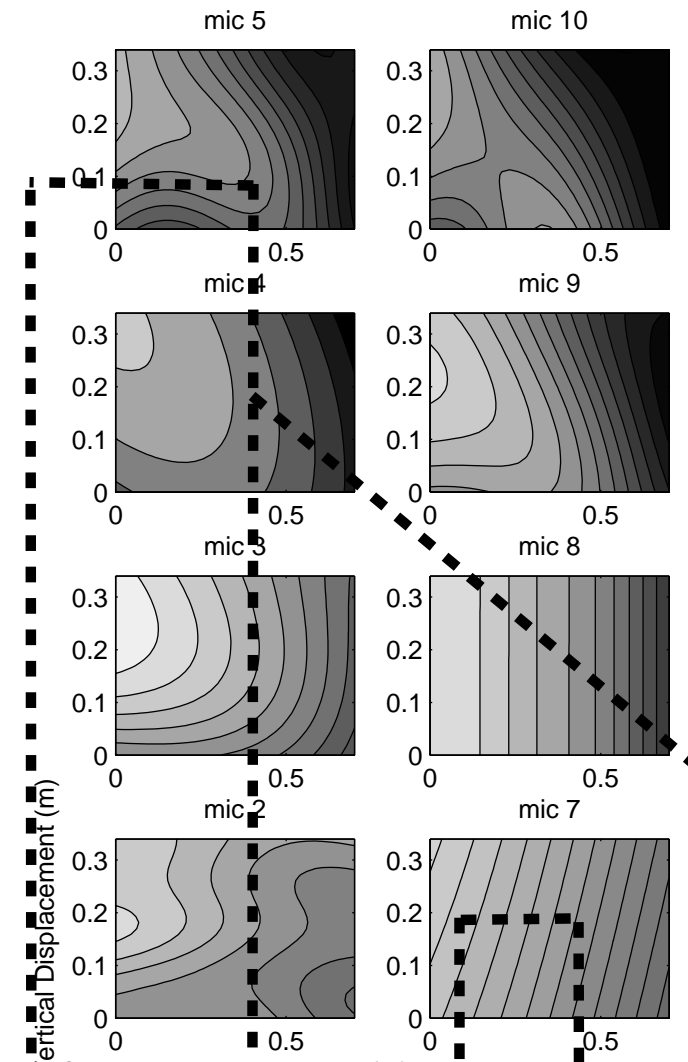

$\stackrel{d}{\infty}$ Streamwise Displacement $(\mathrm{m})$

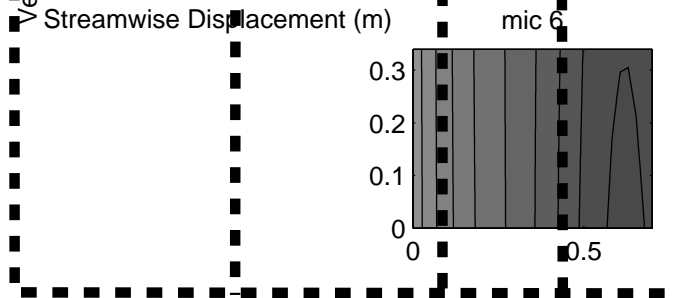

$\operatorname{mic} 15$
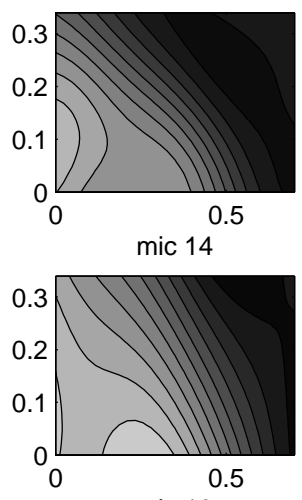

mic 13
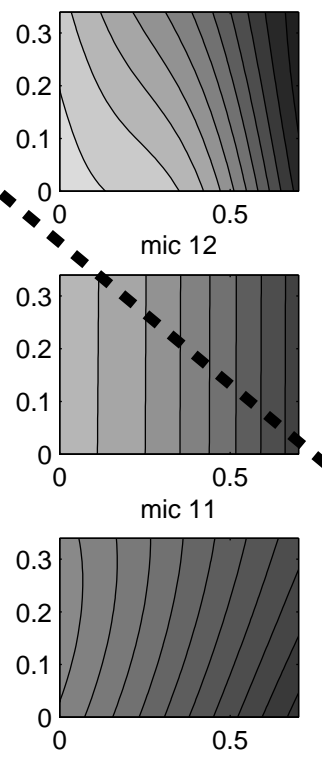
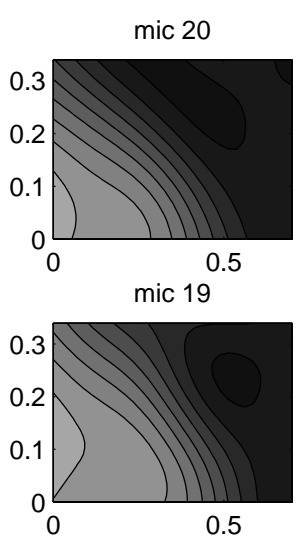

mic 18
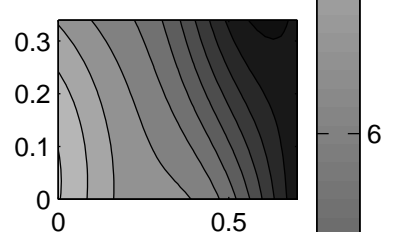

mic 17
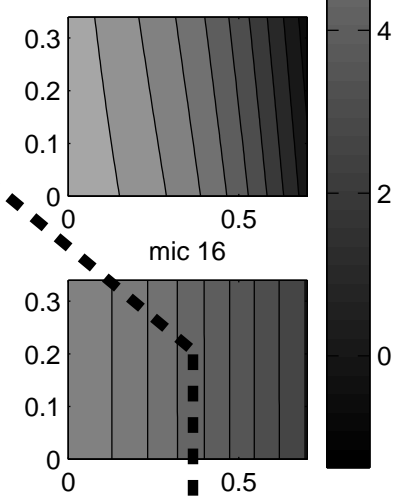

Fig. 11 Kriging prediction of the noise objective in our design space for each microphone, with the dashed lines indicating the position of the aircraft model and nacelle.

one and the rest are given ranks of two or higher (with rank two designs being non-dominated if we were to remove the rank one set, and so on). The GA is then guided to explore through the design space by rewarding or penalising each individual in the population, based on its distance from the Pareto front (encouraging Pareto optimality) and other members (encouraging a uniform spacing of designs along the Pareto front).

From our objectives we require high noise shielding and low $C_{D}$ to obtain a good design. We see that we have conflicting objectives, as the optimal drag occurs at the worst shielding in Fig. 12, and vice versa. This may seem counterintuitive from analysing the surrogates in Figs. 3 and 8 , but 


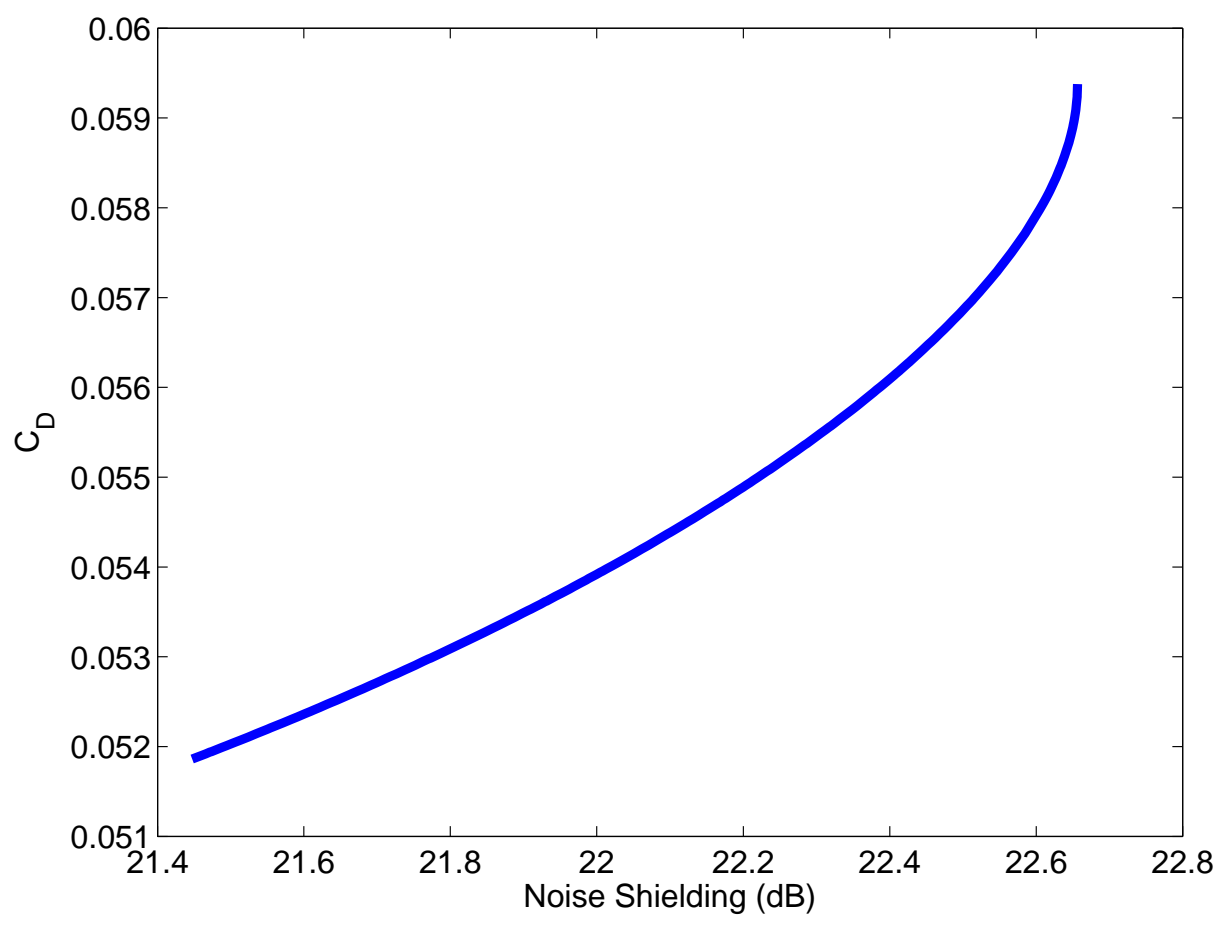

Fig. 12 A Pareto front generated using NSGA II on the $C_{D}$ and noise shielding surrogates shown in Figs. 3 and 8 respectively.

from comparing the axes to the colourbars of our surrogate plots we can see that we are only viewing designs with the engine in its furthest aft position, and with a low vertical displacement. Here the contours of the surrogates are comparably normal to each other, confirming that these objectives are indeed conflicting in this region.

From generating a Pareto front of the non-dominated designs in terms of our noise and drag performance metrics we have a set of designs that a designer can choose to be the most appropriate. This choice may be based on further analysis, typically by evaluating each of these designs from the perspective of a new discipline.

\section{Future Work}

We have presented in this paper a multidisciplinary problem using two objectives. In reality, engineering problems contain many more objectives within a multitude of disciplines - next we intend to add a structural metric to our set of objectives. Another objective that we have not considered here is the change in engine performance when the engine position is moved. We can characterise this by the quality of the flow entering the engine in terms of the pressure profile at the fan face. We also intend to improve the realism of the case study by replacing the flow-through nacelles with nacelles that model the flow entering and leaving the engine.

In this case study we have simplified the problem by characterising it using only two parameters; namely the streamwise and vertical positions of the engine. All of the geometric entities used in this study were designed to be used in a conventional configuration, and had not been modified to 
be used in an engine over-the-wing configuration. Therefore, we cannot quantify from this study if an over-the-wing configuration provides any aerodynamic performance difference compared to the conventional case. We also take note of NASA's approach of propulsion airframe integration in their ERA vehicle systems integration sub-project, where they advocate "Simultaneous wing-nacelle aero shape optimization" 14 to integrate Ultra-High Bypass Ratio (UBH) engines with no interference drag penalty. In that spirit, we intend to fully parameterise this case study to design the wing/pylon/nacelle geometry specifically for the over-the-wing configuration.

\section{Acknowledgements}

We would like to thank the Royal Academy of Engineering for funding the noise shielding experiment, as well as the work of the second author. We are also grateful to John Fithyan and Mathieu Gruber for their help in the experimental setup. We would also like to thank Giles Endicott for his advice on the CFD study, and the Royal Aeronautical Society for the funds made available to the first author to travel to the AIAA Aerospace Sciences Meeting 2010 to present this work.

\section{References}

1 "European Aeronaurtics: A Vision for 2020,," Advisory Council for Aeronautics Research in Europe, 2001.

${ }^{2}$ Crichton, D., Blanco, E., and Law, T. R., "Design and Operation for Ultra Low Noise Take-Off," AIAA 2007-456, 45th AIAA Aerospace Sciences Meeting and Exhibit, 8-11 January, Reno, Navada 2007.

${ }^{3}$ Liebeck, R., "Design of the Blended Wing Body Subsonic Transport," Journal of Aircraft, Vol. 41, No. 1, Jan-Feb 2004, pp. 10-25.

${ }^{4}$ Berton, J. J., Envia, E., and Burley, C. L., "An Analytical Assessment of NASA's N1 Subsonic Fixed Wing Project Noise Goal," 15th AIAA/CEAS Aeroacoustics Conference, 11-13 May 2009.

${ }^{5}$ Berry, D., "The Boeing 777 Engine/Aircraft Integration Aerodynamic Design Process," ICAS-94-6.4.4, 1994, pp. $1305-1320$.

${ }^{6}$ Kinney, D. and Hahn, A.S. Gelhausen, P., "Comparison of Low and High Nacelle Subsonic Transport Configurations," AIAA-97-2318, 1997.

${ }^{7}$ Berton, J., "Noise Reduction Potential of Large, Over-the-Wing Mounted, Advanced Turbofan Engines," Tech. Rep. TM-2000-210025, NASA, 2000.

${ }^{8}$ Ronzheimer, A., Rudnik, R., Broderson, O., and Rossow, C., "Interference Phenomena of Upper-Wing-Mounted Engines," Mitteilung-Deutsche Forschungsanatalt fur Luft und Raumfahrt, 1996, pp. 12.1 - 12.14.

${ }^{9}$ Reshotko, M. and Olsen, W. A., "Preliminary Noise Tests of the Engine-Over-The-Wing Concept II. $10^{\circ}-20^{\circ}$ Flap Position," Tech. Rep. TM X-68104, NASA, 1972.

${ }^{10}$ Jordan, P., "The VFW 614 Fleet," http://www.vfw614.de/flotte_e.html, Accessed Mar 2010.

${ }^{11}$ Fujino, M. and Kawamura, Y., "Wave-Drag Characteristics of an Over-the-Wing Nacelle Business-Jet Configuration," Journal of Aircraft, Vol. 40, 2003, pp. 1177-1184.

${ }^{12}$ Agarwal, A. and Dowling, A., "Low-Frequency Acoustic Shielding by the Silent Aircraft Airframe," AIAA Journal, Vol. 45, No. 2, 2007, pp. 358-365.

${ }^{13}$ Agarwal, A., Dowling, A., Shin, H.-C., and Graham, W., "Ray-Tracing Approach to Calculate Acoustic Shielding by a Flying Wing Airframe," AIAA Journal, Vol. 45, No. 5, 2007, pp. 1080-1090.

${ }^{14}$ Leavitt, L. and Smith, S., "NASA's ERA Vehicle Systems Integration Sub-Project," Royal Aeronautical Society Aerodynamics Conference, 2010.

${ }^{15}$ Thomas, R., Burley, C., and Olson, E., "Recent Progress in Propulsion Airframe Aeroacoustics," Royal Aeronautical Society Aerodynamics Conference, 2010.

${ }^{16}$ Van Zante, D. and Breeze-Stringfellow, A., "The NASA/GE Collaboration on Open Rotor Testing," Royal Aeronautical Society Aerodynamics Conference, 2010. 
${ }^{17}$ Yoneta, R., Sasaki, D., and Nakahashi, K., "Aerodynamic Optimization of an Over-the-Wing-Nacelle-Mount Configuration," AIAA 20101016, 2010.

${ }^{18}$ Forrester, A., Sobester, A., and Keane, A., Engineering Design via Surrogate Modelling, Wiley, 2008.

${ }^{19}$ Leary, S., Bhaskar, A., and Keane, A., "Optimal orthogonal-array-based latin hypercubes," Journal of Applied Statistics, Vol. 30, No. 5, 2003, pp. 585-598.

${ }^{20}$ Tang, B., "Orthogonal Array-Based Latin Hypercubes," Journal of the American Statistical Association, Vol. 88(424), 1993, pp. 1392-1397.

${ }^{21}$ Laflin, K., Klausmeyer, S., Zickuhr, T., Vassberg, J., Wahls, R., Morrison, J., Broderson, O., Rakowitz, M., Tinoco, E., and Godard, J., "Data Summary from the Second AIAA Computational Fluid Dynamics Drag Prediction Workshop," Journal of Aircraft, Vol. 42, No. 5, 2005, pp. 1165-1178.

${ }^{22}$ Harpoon Website, http://www.sharc.co.uk/index.htm.

${ }^{23}$ Fluent Inc, Fluent User's guide.

${ }^{24}$ Vassberg, J., Tinoco, E., Mani, M., Broderson, O., Eisfeld, B., Wahls, R., Morrison, J., Zickuhr, T., Laflin, K., and Mavriplis, D., "Summary of the Third AIAA Drag Prediction Workshop," AIAA 2007-0260, 2007.

${ }^{25}$ Lowis, C., Joseph, P., and Kempton, A., "Estimation of the far-field directivity of broadband aeroengine fan noise using an in-duct axial microphone array," Journal of Sound and Vibration, Vol. 329, No. 19, 2010, pp. 3940-3957.

${ }^{26}$ Deb, K., Pratap, A., Agarwal, S., and Meyarivan, T., "A fast and elitist multiobjective genetic algorithm: NSGAII," IEEE Transactions on Evolutionary Computation, Vol. 6, No. 2, 2002, pp. 182-197. 